\title{
POUR UNE HISTOIRE CONCEPTUELLE DU POLITIQUE (NOTE DE TRAVAIL *)
}

L'historien des idées, le philosophe et l'historien des faits et des institutions se sont pendant longtemps partagé l'appréhension du champ politique, le découpant en trois territoires disciplinaires strictement cloisonnés. Les héritiers de Langlois-Seignobos et de Fustel de Coulanges, les émules d'Émile Faguet et les descendants de Victor Cousin ont tranquillement cultivé, pendant plus d'un demi-siècle, leurs petits jardins de " spécialistes " universitaires sans même songer à jeter un regard sur ceux de leurs voisins. Et cela quelles que soient d'ailleurs les préférences partisanes et les orientations philosophiques dont étaient empreints leurs travaux. D'où l'usure progressive de ces genres traditionnels. Pour ce qui les concerne, les plus doués des jeunes historiens ont clairement pris leurs distances dès les années 1930. On connaît assez les termes de leur critique pour ne pas avoir à y revenir. Dans sa contribution à Faire de l'histoire ', Jacques Julliard explique à juste titre de cette manière la mauvaise presse dont l'histoire politique jouit chez les historiens français depuis une quarantaine d'années.

Est-ce à dire que l'étude du politique ait été progressivement abandonnée, cédant complètement la place aux intérêts nouveaux pour l'économique, le social ou le culturel investis par la génération des Ariès, des Braudel et des Febvre ? Les choses ne se sont pas vraiment passées ainsi. Le déclin de l'histoire politique traditionnelle s'est aussi accompagné du développement de l'histoire des mentalités politiques et surtout de la sociologie politique. Dans La République au village ${ }^{2}$, Maurice Agulhon a donné ses lettres de noblesse à la première, cherchant à frayer la voie d'une nouvelle approche de l'histoire politique soucieuse de maintenir ses distances vis-à-vis des problématiques déterministes (cf. Ernest Labrousse : " Le social retarde sur l'économique, et le mental retarde sur le social ") et des strictes descriptions ethnologiques. La sociologie

\footnotetext{
* Sur le sens de cette note de travail, voir p. 105.

1. Paris, Gallimard, 1974, t. 2, "Nouvelles approches ".

2. Paris, Le Seuil, 1979 (1 re $^{\text {re }}$., Plon, 1970).
} 
politique, de son côté, a connu une indéniable faveur, la création d'une agrégation de sciences politiques consacrant au milieu des années 1970 sa spécificité universitaire. Outre le regain d'intérêt pour l'histoire des idées qui en est résulté, le développement de cette discipline s'est surtout traduit par la multiplication des travaux sur les forces politiques et le système politique. S'engouffrant dans la voie ouverte au début du siècle par Roberto Michels ${ }^{3}$ et Moisei Ostrogorski ${ }^{4}$, Annie Kriegel ${ }^{5}$, Maurice Duverger ${ }^{6}$ et Georges Lavau ${ }^{7}$, pour ne citer que quelques noms, ont consacré des livres, devenus classiques, au PCF ou à l'étude globale des partis. Les travaux sur les élites politiques et le fonctionnement général du système politique se sont par ailleurs multipliés.

Bien des livres publiés dans ces deux directions de l'histoire des mentalités politiques et de la sociologie politique ont innové et permis de renouveler l'approche du champ politique. Mais ils n'ont pas comblé le vide laissé par le dépérissement progressif de l'histoire des idées et de l'histoire des institutions. Le déplacement de méthode et d'objet qu'ils ont opéré a également rapidement marqué ses limites. On le voit bien en prenant l'exemple du fait communiste. La multiplication des études sur le PCF ou le mouvement communiste international, et il en est d'excellentes, n'a pas vraiment permis de mieux saisir l'essence du totalitarisme, limitant par-là même la compréhension que l'on pouvait avoir du fonctionnement des sociétés communistes. D'où l'intérêt croissant pour la philosophie politique depuis le début des années $1970^{8}$.

L'approche des problèmes politiques a ainsi été marquée depuis trente ans par une série de déplacements successifs, soit tout simplement par glissement à l'intérieur même de la profession des historiens, soit par des modes de réactivation disciplinaire. Les historiens des mentalités ont succédé aux historiens dits événementiels d'un côté. Les sociologues ont pris le relais des historiens, puis les philosophes celui des sociologues de l'autre. Une première discussion pourrait déjà prendre place sur la base de ce constat pour tenter d'évaluer l'apport respectif des grands ouvrages qui ont caractérisé chacune de ces étapes. Je ne suis pas sûr qu'elle ait vraiment un grand intérêt, si la question est posée dans ces termes. Je

3. Les Partis politiques (1911), Paris, Flammarion, 1971.

4. La Démocratie et les partis politiques (2 vol., 1902), rééd. abrégée par P. RoSANVALLON, Paris, Le Seuil, 1979.

5. Cf. en particulier, Les Communistes français. Essai d'ethnographie politique, Paris, Le Seuil, 1968.

6. Les Partis politiques, Paris, A. Colin, 1951.

7. À quoi sert le parti communiste français?, Paris, Fayard, 1982.

8. Le lancement de nouvelles collections comme " Les classiques de la politique " chez Garnier, "Critique de la politique " chez Payot, " Recherches politiques " aux P.U.F. ou de revues comme Libre puis Passé-Présent en sont des signes parmi d'autres. 
m'en rends d'autant mieux compte que j'ai moi-même été tenté un moment de penser dans cette perspective la rénovation de l'étude du politique (en faisant naturellement de la philosophie politique la nouvelle voie royale d'accès au politique, supposé opérer par rapport aux sociologues et aux historiens des mentalités une rupture équivalente à celle que les pères fondateurs des Annales avaient provoqué vis-à-vis de leurs prédécesseurs). Que l'apport de la philosophie politique soit décisif et incontournable, je n'en doute évidemment pas. De même suis-je intimement persuadé qu'elle a ouvert des horizons nouveaux à la compréhension du monde contemporain. L'arrivée en force de la philosophie politique sur le devant de la scène intellectuelle française des années 1980 est certes un phénomène majeur, qui s'explique bien par un certain épuisement des grands courants ayant marqué le développement des sciences sociales dans les années 1960. Mais il ne se confond pas avec un autre phénomène, plus diffus, connexe mais pourtant distinct : la formation progressive d'une histoire conceptuelle du politique, dépassant le jeu des clivages et des déplacements que je viens d'évoquer.

Le fait intellectuellement le plus intéressant de ces dix dernières années me semble résider dans le rapprochement progressif des problématiques d'analyse du politique de spécialistes issus de disciplines différentes. Tout un ensemble de réorientations disciplinaires, autonomes au départ, ont peu à peu dessiné un lieu commun. Citons à titre indicatif pour illustrer le propos :

- la redécouverte et la rénovation de l'histoire des idées avec des travaux come ceux de C. Nicolet (Histoire de l'idée républicaine en France ${ }^{9}$ ), de P. Manent (Naissance de la politique moderne ${ }^{10}$ ), de P. Bénichou (Le Sacre de l'écrivain ") ou de L. Dumont (Homo Aequalis ${ }^{12}$ ) ;

- la rénovation philosophique de l'histoire politique avec les livres de

F. Furet (Penser la Révolution française ${ }^{13}$ ) ou de B. Baczko (Lumières de l'utopie $\left.{ }^{14}\right)$;

- la philosophie politique de l'événement avec les essais de C. Lefort rassemblés dans $L$ 'Invention démocratique ${ }^{15}$;

- le développement d'une anthropologie politique dans la lignée des

9. Paris, Gallimard, 1982.

10. Paris, Payot, 1977.

11. Paris, José Corti, 1973.

12. Paris, Gallimard, 1976.

13. Paris, Gallimard, 1978.

14. Paris, Payot, 1978.

15. Paris, Fayard, 1981. 
ouvrages de P. Clastres (La Société contre l'État ${ }^{16}$ ) et de M. Gauchet et G. Swain (La Pratique de l'esprit humain ${ }^{17}$ ) ;

- la réactivation de la philosophie du droit par des jeunes philosophes comme L. Ferry et A. Renaut (Des droits de l'homme à l'idée républicaine ${ }^{18}$ ).

Tous ces ouvrages ont en commun une certaine dimension philosophique. Mais ce trait ne suffit pas à caractériser leur air de famille. L'unité de ces travaux réside plus précisément dans un présupposé méthodologique et dans une question. Le présupposé méthodologique tient à la définition implicite du politique sur laquelle ils se fondent. Le politique n'est pas pour eux une " instance » ou un " domaine " parmi d'autres de la réalité : il est le lieu où s'articulent le social et sa représentation, la matrice symbolique dans laquelle l'expérience collective s'enracine et se réfléchit à la fois. La question ? C'est celle de la modernité, de son avènement et de son travail.

Le temps me semble maintenant venu de dépasser le simple constat de cette convergence très globalement définie pour construire de façon plus rigoureuse la notion d'histoire conceptuelle du politique dont ces différents travaux participent. Le premier pas de cette construction implique de bien différencier cette histoire conceptuelle de l'histoire traditionnelle des idées. Différenciation d'autant plus nécessaire que l'on sent bien que les auteurs et les ouvrages que nous venons de citer donnent assez souvent l'impression d'être sur le fil du rasoir - position précaire que je ressens naturellement aussi comme étant la mienne.

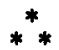

L'histoire traditionnelle des idées me semble marquée par un certain nombre de faiblesses méthodologiques qu'il faut soigneusement répertorier. On peut au moins en noter cinq :

1) La tentation du dictionnaire. Considérons des ouvrages classiques de la fin du XIX siècle comme ceux de Émile Faguet, Politiques et moralistes au XIX $X^{e}$ siècle ${ }^{19}$ ou de Paul Janet, Histoire de la philosophie morale et politique ${ }^{20}$. Ils ne sont, en fait, constitués que d'une addition de monographies consacrées à des auteurs. Chacune de ces études peut avoir des mérites intrinsèques mais leur regroupement ne compose pas à

16. Paris, Ed. de Minuit, 1974.

17. Paris, Gallimard, 1980.

18. Paris, P.U.F., 1985.

19. Paris, s.d., 3 vol.

20. Paris, 1858,2 vol. 
proprement parler un ouvrage permettant de comprendre le mouvement intellectuel du siècle. On peut adresser le même reproche à des livres plus récents, comme celui de Pierre Mesnard, $L$ 'Essor de la philosophie politique au seizième siècle ${ }^{21}$. Si je n'ai pas lu Althusius ou Hotman, cet ouvrage me donnera une idée de leur cuvre, mais la présentation successive d'une vingtaine de grands auteurs de l'époque ne fournit pas les clefs du basculement d'ensemble qui s'opère alors dans la philosophie politique. Le Temps des prophètes ${ }^{22}$ de Paul Bénichou ou l'Histoire des idéologies ${ }^{23}$ publiée sous la direction de François Châtelet ont le même inconvénient. Cela ne signifie pas que ces ouvrages doivent être rejetés. La plupart de ceux que nous avons cités constituent au contraire de précieux instruments de travail. Mais ils n'ont rien d'historique. Ce sont en fait des dictionnaires spécialisés d'œuvres ou des manuels de doctrines politiques. Ils peuvent être remarquables dans le détail de leurs analyses, fournir une mine d'utiles indications bibliographiques, présenter une habile synthèse de telle ou telle œuvre, voire renouveler l'appréhension d'un auteur particulier, mais ils ne sont généralement soutenus par aucune problématique globale. Ce sont des livres faits pour être consultés, ils n'ont pas besoin d'être lus de façon continue pour livrer leur apport. On les range dans sa bibliothèque sur les rayons des usuels, à côté des dictionnaires. C'est déjà une place de choix.

2) L'histoire des doctrines. Soit au départ une « doctrine " quelconque : l'idée de progrès, le socialisme, le libéralisme, le contrat social, l'utilitarisme, etc. L' " histoire » de cette doctrine consiste généralement en un laborieux travail de repérage du cheminement de l' "idée " dans l'histoire. Exemplaires de ce genre, des ouvrages comme ceux d'E. Halévy, La Formation du radicalisme philosophique ${ }^{24}$, de R. Derathé, J.-J. Rousseau et la science politique de son temps ${ }^{25}$, d'A. Lichtenberger, Le Socialisme au XVIII siècle ${ }^{26}$. Ils présupposent, en fait, que la doctrine qu'ils étudient représente quelque chose d'abouti et de stable. Conception ouvertement finaliste qui fait de ces ouvrages de longues traques de précurseurs. On part de Rousseau, de Bentham ou de Marx pour repérer tous ceux qui les « annoncent ", les « préfigurent ", ou " marquent une étape " dans la formation de la doctrine qu'ils incarnent. Le lecteur voit défiler devant lui des chapitres dans les-

21. Paris, Vrin, 1969 (1re éd. 1936).

22. Paris, Gallimard, 1977.

23. Paris, Hachette, 1978, 3 vol.

24. Paris, 1901-1904, 3 vol.

25. Paris, Vrin, 1974 (1re éd. 1950).

26. Paris, 1895. 
quels des œuvres ne sont interrogées que par rapport à un point d'arrivée déjà connu. Dans leur forme savante (R. Derathé, E. Halévy), de telles histoires ont au moins le mérite de rassembler un vrai matériau documentaire et elles ont la prudence de se donner des bornes assez strictes dans leur recherche des anticipations de la doctrine qu'elles étudient. Dans leur forme vulgaire, elles tendent inévitablement à élargir leur champ, pour faire de la doctrine considérée l'aboutissement radieux de toute l'histoire philosophique. Il est, par exemple, des histoires du matérialisme historique qui, partant des philosophes de l'Antiquité, dressent laborieusement la longue liste de tous ceux qui ont " aperçu ", tout en la manquant généralement, ladite doctrine jusqu'à ce qu'elle soit enfin saisie dans toute son intégrité par Marx. Les doctrines sont comme des germes dont on contemple la croissance dans des ouvres qui n'ont au fond aucun autre intérêt que d'en refléter le cheminement tortueux. Une telle histoire n'a paradoxalement rien d'historique. C'est la notion même de doctrine qui est ici en cause.

3) Le comparatisme textuel. Il cohabite souvent avec l'histoire des doctrines. Il consiste à ne penser une œuvre que par rapport à celles qui la suivent ou la précèdent, à ne la faire exister que relativement à ce qui lui est extérieur. Que de présentations de Machiavel, Auguste Comte ou Locke dans lesquelles il est surtout question de ce que ne sont pas leurs œuvres, des ouvrages auxquels ils empruntent, des travaux futurs auxquels ils ouvrent la voie. L'histoire des idées consiste alors à manipuler une sorte de kaléidoscope grâce auquel on peut arranger une multitude de figures toujours bien ordonnées. Ce comparatisme systématique dissimule souvent une absence totale de capacité d'interrogation des textes. L'art du commentaire revient à s'abriter en permanence derrière la présupposition d'un caractère explicatif en lui-même de la référence. Dire de Sieyès qu'il annonce Benjamin Constant n'apprend évidemment rien si l'on sait seulement que ce dernier annonce Tocqueville ou qu'il s'oppose à Joseph de Maistre. L'histoire des idées procède pourtant souvent de la sorte. Ce comparatisme généralisé marque généralement une indigence de la réflexion qui se cache derrière un exercice de pseudoérudition. À ce défaut de base s'ajoute, en outre, dans la plupart des cas, un aveuglement sur les différences de contexte dans lesquels les œuvres prennent sens. On compare Adam Smith et Benjamin Constant comme si leurs œuvres cherchaient à répondre à la même question. L'œuvre est implicitement appréhendée comme un texte autonome, elle n'est jamais conçue comme un travail dont il s'agirait de comprendre les déterminants. Il en résulte d'ailleurs généralement une accumulation d'équivoques, les mots eux-mêmes étant supposés ne pas avoir d'histoire. On comparera Rousseau, Tocqueville et Gambetta sans se soucier 
du fait que le terme de démocratie n'a pas le même sens pour chacun d'eux.

4) Le reconstructivisme. L'analyse et le commentaire ont dans ce cas pour objet pratique de récrire une œuvre pour l'établir dans une cohérence et une clarté qui est censée faire défaut à l'auteur. Le Marx d'Althusser est l'exemple presque trop parfait de ce reconstructivisme. Mais chacun peut aussi avoir son Burke, son Machiavel ou son Tocqueville. C'est une manière de penser par procuration, à l'abri d'une œuvre sur laquelle on peut à la limite projeter n'importe quoi. L'œuvre n'est au fond pas prise au sérieux, elle n'est que le support d'une interprétation qui l'étouffe et l'envahit, elle reste regardée à distance.

5) Le typologisme. Il sévit surtout dans les manuels, comme l'Histoire des idées politiques de Jean Touchard (Thémis) ou l'ouvrage de Marcel Prélot portant le même titre (Dalloz), qui constituent malheureusement encore la base du gavage universitaire dans les facultés de droit et de sciences politiques. L'histoire des idées se réduit dans ces ouvrages à la confection d'une sorte de vaste catalogue des écoles de pensée et des doctrines. Les auteurs distribuent quelques centaines d'œuvres dans des petits tiroirs drôlement étiquetés. Ouvrons le Prélot. On apprend à y distinguer le nationalisme « émotif » (Barrès, Péguy), " intégral » (Maurras), " totalitaire " (Hitler, Mussolini), " personnalisé » (de Gaulle). Quant au libéralisme, il peut être "pur " (Constant), doctrinaire, démocratique, catholique, constructif, extrêmiste ; le terme de libéralisme n'étant d'ailleurs jamais défini. Il n'y a aucune histoire dans ces manuels qui ont une propension à tout envisager en termes d'écoles, d'étapes, de périodes, de courants. L'art de la classification se substitue ici à la pensée et à la compréhension des œuvres. Je ne pense pas que l'on puisse comprendre quoi que ce soit à Benjamin Constant en le qualifiant de libéral pur, pas plus que le fait de savoir que Saint-Simon est un socialiste utopique puisse être d'un grand secours pour quiconque.

Ces cinq faiblesses de l'histoire des idées se mêlent souvent à des degrés divers. Le problème, notons-le, ne se limite pas au seul débat texte/contexte tel qu'il s'est par exemple déroulé en Angleterre et aux États-Unis au début des années 1970. Le plus gros handicap de tous ces ouvrages traditionnels d'histoire des idées est qu'ils ne nous font rien comprendre d'historique même lorsqu'ils nous apprennent beaucoup d'autres choses. C'est d'abord en fonction de cette limite fondamentale qu'il faut considérer l'apport de l'histoire conceptuelle du politique.

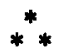

L'objet de l'histoire conceptuelle du politique est de comprendre la formation et l'évolution des rationalités politiques, c'est-à-dire des 
systèmes de représentations qui commandent la façon dont une époque, un pays ou des groupes sociaux conduisent leur action et envisagent leur avenir. Partant de l'idée que ces représentations ne sont pas un englobant extérieur à la conscience des acteurs - comme le sont par exemple les mentalités -, mais qu'elles résultent au contraire d'un travail permanent de réflexion de la société sur elle-même, elle a pour but 1) de faire l'histoire de la manière dont une époque, un pays ou des groupes sociaux cherchent à construire des réponses à ce qu'ils perçoivent plus ou moins confusément comme un problème et 2) de faire l'histoire du travail opéré par l'interaction permanente entre la réalité et sa représentation en définissant des champs historico-problématiques. Son objet est ainsi d'identifier les " nœuds historiques " autour desquels de nouvelles rationalités politiques et sociales s'organisent, des représentations du politique se modifient en rapport avec les transformations dans les institutions, les techniques de gestion et les formes du rapport social. Elle est histoire politique dans la mesure où la sphère du politique est le lieu d'articulation du social et de sa représentation. Elle est histoire conceptuelle parce que c'est autour de concepts - l'égalité, la souveraineté, la démocratie, etc. - que se nouent et s'éprouvent l'intelligibilité des situations et le principe de leur activation.

Quelques exemples de ces nœuds et de ces questions : comment travaille la question « terminer la Révolution » dans la culture politique du $\mathrm{XIX}^{\mathrm{e}}$ siècle dans son rapport à la perception de Thermidor ? Comment la question du libéralisme et de la démocratie se noue-t-elle pendant la Révolution ? Comment et pourquoi la pensée du lien social se transforme-t-elle dans l'Angleterre du xville siècle ? Ces exemples ponctuels nous amènent à faire une remarque fondamentale : l'histoire conceptuelle du politique, si elle peut appréhender quantité d'objets distincts, est néanmoins toujours rapportée à une perspective centrale, celle de l'interrogation sur le sens de la modernité politique, de son avènement et de son développement ; modernité politique liée à l'émergence progressive de l'individu comme figure génératrice du social, mettant la question des rapports entre le libéralisme et la démocratie au cœur de la dynamique de l'évolution des sociétés.

Contrairement à l'histoire des idées, la matière de cette histoire conceptuelle du politique ne peut se limiter à l'analyse et au commentaire des grandes œuvres, même si celles-ci peuvent dans certains cas être considérées à juste titre comme des pôles cristallisant les questions qu'une époque se pose et les réponses qu'elle tente d'y apporter. Elle emprunte à l'histoire des mentalités ${ }^{27}$ le souci d'incorporer l'ensemble des élé-

27. Je renvoie sur ce point au numéro spécial « Histoire des sciences et mentalités », 
ments qui composent cet objet complexe qu'est une culture politique : le mode de lecture des grandes œuvres théoriques, les œuvres littéraires, la presse et les mouvements d'opinion, les pamphlets et les discours de circonstance, les emblèmes et les signes. On ne peut par exemple se contenter d'appréhender la question des rapports libéralisme/démocratie pendant la Révolution française en supposant qu'elle consiste en une sorte de débat au sommet entre Rousseau et Montesquieu. Il faut prendre la peine de saisir ce qu'avaient retenu de ces auteurs ceux qui s'en réclament, interroger la masse des pétitions envoyées à l'Assemblée, se plonger dans l'univers des brochures et des libelles, relire les débats parlementaires, pénétrer dans les clubs et les commissions. Il faut également faire l'histoire des mots et étudier l'évolution de la langue (on n'entend pas la même chose en 1789 ou en 1793 quand on parle de démocratie par exemple). Plus largement encore, l'histoire des événements et des institutions doit être prise en compte de façon permanente. Il n'y a pas, dans cette mesure, de matière propre à l'histoire conceptuelle : elle consiste d'abord à collecter l'ensemble des matériaux sur lesquels s'appuient, de façon séparée, les historiens des idées, des mentalités, des institutions et des événements.

Son originalité réside plus dans sa méthode que dans sa matière. Cette méthode est à la fois interactive et compréhensive. Interactive car elle consiste à analyser la façon dont une culture politique, des institutions et des événements travaillent les uns dans les autres, composant des figures plus ou moins stables : analyse des plis, des écarts, des recouvrements, des convergences, des vides qui accompagnent ce travail et en marquent les équivoques ou les ambiguïtés comme les formes d'accomplissement. Compréhensive car elle s'efforce de saisir une question en la resituant dans ses conditions effectives d'émergence. Il est impossible dans ces conditions de s'en tenir à une approche « objectiviste » qui présuppose de la part de l'historien qu'il surplombe et maîtrise de l'extérieur un objet inerte. L'approche compréhensive cherche à appréhender l'histoire en train de se faire, alors qu'elle est encore possibilité, avant qu'elle ne soit établie dans son statut passif de nécessité. Comprendre au sens de Max Weber (verstehen) dans le champ historique implique de reconstruire la façon dont des acteurs élaborent leur intelligence des situations, de repérer les récusations et les attractions à partir desquelles ils pensent leur action, de dessiner l'arbre des impasses et des possibilités

Revue de synthèse, 111-112, juil.-déc. 1983, et en particulier à l'article de Roger CHARTIER, " Histoire intellectuelle et histoire des mentalités. Trajectoires et questions ", qui plaide à juste titre pour un rapprochement de l'histoire des idées et de l'histoire des mentalités. 
qui structure implicitement leur horizon. Méthode empathique à cet égard par ce qu'elle suppose de capacité à reprendre une question en se situant à l'intérieur de son travail. Mais empathie naturellement limitée par la prise de distance qui permet de penser les zones d'aveuglement et les contradictions des acteurs ou des auteurs. Empathie contrôlée, si l'on veut 28.

Cette approche compréhensive tire sa justification de la présupposition d'un invariant entre la situation de l'auteur ou de l'acteur étudiés et la nôtre. Pour le sociologue wébérien, cet invariant est celui de la nature humaine. Dans le cas de l'histoire conceptuelle des idées, il consiste dans la conscience que nous avons de continuer à être immergés dans les questions dont le travail est étudié. L'œuvre de l'historien peut ainsi ouvrir la voie à un engagement intellectuel de type nouveau. Celui-ci ne consiste pas à investir des idées, des préférences ou des a priori dans une lecture ou un exposé ; pas plus qu'il n'est la simple mise en scène des groupes sociaux ou des auteurs vers lesquels une sympathie spontanée peut porter l'interprète. Le but est de faire de cette histoire conceptuelle une ressource de compréhension du présent. Idée extrêmement banale dira-t-on à juste titre : l'histoire du passé a toujours pour intérêt d'éclairer le présent. $\grave{A}$ y regarder de près, les choses ne sont pourtant pas aussi simples. Bien des livres d'histoire cherchent, en effet, plutôt à réinterpréter le passé en fonction du présent ou même de l'avenir tel qu'on l'imagine. Cette inversion des termes de l'opération de compréhension me semble particulièrement frappante dans le domaine de l'histoire politique. Prenons l'exemple de l'histoire politique de la Révolution française. Le livre d'Aulard ${ }^{29}$, qui constitue l'ouvrage classique de référence sur le sujet, analyse le mouvement politique de la Révolution en rapportant en permanence les discours et les institutions politiques de la période à ce qu'il estime être la forme stable et achevée de l'idée

28. L'empathie, contrairement à la sympathie, n'implique aucune identification. Cette distinction élémentaire n'a pas l'air d'être toujours comprise. Je m'en suis rendu compte lorsque je me suis vu reprocher par certains d'avoir écrit sur le libéralisme du début du $\mathrm{XIX}^{\mathbf{c}}$ siècle en partant de Guizot. L'empathie nécessite à la fois un travail d'information (qui me rende capable d'évaluer les données de la situation dans laquelle s'est trouvé un auteur et de bien saisir la structure du champ historico-intellectuel dans lequel il s'est mû) et un travail de distanciation (qui me fasse évaluer en permanence la différence entre ma situation propre et celle de l'observé). P.-H. Maucorps et R. Bassoul écrivent dans Empathie et connaissance d'autrui, cité in Jean FoulquIÉ, Dictionnaire de la langue philosophique, Paris, P.U.F., 1969 : « L'empathie présente un caractère plus désintéressé, plus conjectural et en quelque sorte plus spéculatif que la sympathie. Aussi apparaît-elle essentiellement comme une démarche participative visant à la compréhension d'autrui en tant qu'autrui et à la prévision de ses potentialités. "

29. Histoire politique de la révolution française, Paris, 1913. 
démocratique ${ }^{30}$. Il suit ainsi les avancées et les reculs de la démocratie de 1789 à 1799 à partir de sa vision propre de la démocratie (le gouvernement pour le peuple et par le suffrage universel). Il juge cette période à partir du présent pris comme point fixe. Une histoire gradualiste et linéaire de cette sorte considère ainsi comme un donné et un acquis certain (le suffrage universel = la démocratie) ce qui est, en fait, le lieu de travail d'un problème (la réduction progressive de l'idée démocratique à celle de vote). Aulard fait comme si l'idée démocratique était présente au départ, n'étant empêchée de se réaliser pleinement que par les circonstances, l'insuffisant discernement des acteurs ou les données de la lutte des classes entre le peuple et la bourgeoisie. L'histoire comprise de cette façon est toujours simple : elle est le lieu où s'affrontent des forces contraires (l'action et la réaction, le progressiste et le rétrograde, le moderne et l'archaïque, le bourgeois et le populaire) dont la résultante explique les avancées et les reculs de l'idée. Le passé est jugé du point de vue d'un présent qui n'est pas lui-même pensé. L'histoire devient dans ces conditions un véritable obstacle à la compréhension du présent. L'histoire conceptuelle du politique, dans sa dimension compréhensive, permet au contraire de supprimer la barrière qui sépare l'histoire politique de la philosophie politique. Compréhension du passé et interrogation sur le présent participent d'une même démarche intellectuelle. Elle offre d'ailleurs par-là même un terrain de rencontre à l'essayisme et à l'érudition que l'on présente volontiers comme antagoniques. L'érudition est la condition indispensable de l'appréhension du travail qui s'opère dans l'histoire (la somme des informations qu'il faut mobiliser et des lectures qu'il faut opérer est, en effet, considérable pour effectuer une démarche compréhensive) et l'essayisme, comme forme d'intervention dans l'actualité, est le moteur de l'interrogation qui fonde le désir de connaître et de comprendre.

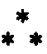

Ces quelques remarques, volontairement esquissées à gros traits, suggèrent que l'histoire conceptuelle du politique ne conduit pas tant à rejeter les voies traditionnelles de l'histoire des idées et de l'histoire des événements et des institutions, ou celles plus récentes de l'histoire des mentalités, qu'à ressaisir leur matière dans une perspective différente. Travail de ressaisissement qui peut expliquer dans certains cas le risque d'un

30. Le sous-titre du livre d'Aulard - «Origines et développement de la démocratie et de la république " - est en lui-même une illustration de cette conception. 
simple retour. C'est particulièrement visible en matière d'histoire des idées. Ce champ a, en effet, été si longtemps déserté qu'il faut souvent en reconstruire la matière la plus traditionnelle avant de la faire travailler dans la perspective de l'histoire conceptuelle. Double effort de rattrapage et d'innovation qu'il faut, par la force des choses, mener de front.

L'histoire conceptuelle ainsi définie doit, enfin, encore être distinguée des quelques tentatives qui ont récemment été faites pour rénover l'histoire des idées : celle de l'histoire contextuelle des idées (cf. les travaux de Quentin Skinner) en particulier. Skinner, l'auteur du très beau The Foundations of Modern Political Thought ${ }^{31}$, a cherché à dépasser l'antagonisme, particulièrement marqué dans les pays anglo-saxons, entre la lecture " philosophique " des grands auteurs ${ }^{32}$, fondée sur l'érection du texte en objet clos et autosuffisant ${ }^{33}$, et la lecture « historique " qui, imprégnée de relents de marxisme, tend à faire des œuvres de simples produits idéologiques dérivés des circonstances et déterminés par elles. Fortement marqué par les travaux de J. L. Austin ${ }^{34}$, Skinner, outre son souci de ne pas s'en tenir aux " grands auteurs ", a cherché à lire les textes comme des "actes linguistiques " situés dans des " champs de signification conventionnellement reconnaissables". Le texte est lu comme un discours dont la pointe ne peut être comprise que si l'on resitue les intentions de l'auteur dans un contexte de conventions. Approche qui a beaucoup renouvelé l'histoire des idées et permis d'engager un dialogue entre l'historien et le philosophe, mais dont le caractère innovateur a été, me semble-t-il, limité par la non-distinction de la problématique des " problèmes éternels de la philosophie " et de celle du travail des questions. Les termes dans lesquels le débat méthodologique sur la façon de faire de l'histoire des idées a été mené aux

31. Cambridge University Press, 1978, 2 vol. On peut le comparer à l'ouvrage cité de P. Mesnard pour mesurer l'abîme qui sépare les deux livres. Skinner a expliqué sa méthode dans une série de très intéressants articles. Citons en particulier : « Meaning and Understanding in the History of Ideas ", History and Theory, VII, 1, 1969 ; "Some Problems in the Analysis of Political Thought and Action ", Political Theory, vol. 2, 3, August 1974 (l'ensemble du numéro reprend les actes d'un symposium consacré à Skinner) ; " Motives, Intentions and the Interpretation of Texts ", New Literary History, vol. 3, 1972.

32. Cf. en particulier comme représentants de la " text school ", Leo StRAuss et J. CROPSEY qui résument bien leur point de vue dans leur History of Political Philosophy, Chicago University Press, 1963.

33. J'ai récemment rencontré un jeune étudiant de Chicago auquel le professeur, qui est un disciple connu de $\mathbf{L}$. Strauss, avait donné un devoir à rédiger sur la Théorie des sentiments moraux d'A. Smith en lui interdisant de lire aucun ouvrage de commentaire ou aucun livre d'histoire sur la période !

34. Cf. John Langshaw Austin, How to Do Things with Words, Oxford, Clarendon Press, 1962. Pour Austin, rappelons-le, le langage est une activité qui accomplit quelque chose, il n'est pas seulement un opérateur passif de la signification. 
Etats-Unis et en Angleterre ${ }^{35}$ ont, en effet, conduit Skinner à soupçonner trop systématiquement de " philosophia perennis » tout ce qui tendait intellectuellement à articuler la lecture des questions du présent avec celle du passé ${ }^{36}$. Les conditions dans lesquelles il a développé sa critique de l'histoire traditionnelle des idées l'ont mené à ne pas franchir le pas qui l'aurait très naturellement poussé à prolonger sa démarche vers l'histoire conceptuelle du politique : il a voulu, ou il a dû, rester dans son rôle de professeur à Cambridge. Son apport reste pourtant incontournable et je reconnais ma dette à son égard.

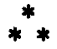

L'histoire conceptuelle du politique ne se fonde pas sur l'application de recettes qu'il suffirait d'appliquer mécaniquement pour rédiger un livre qui puisse illustrer, mieux que ne saurait le faire une nécessairement maladroite " déclaration d'intention ", ce qu'elle aspire à faire. Toute œuvre reste une tentative fragile de produire un supplément d'intelligibilité par l'écriture. Là peut-être encore plus qu'ailleurs.

Pierre Rosanvallon, E.H.E.S.S.

* Le statut de note de travail de ce texte est destiné à en préserver le caractère ouvert. La réflexion épistémologique et programmatique qu'il développe s'insère, en effet, dans une période de rédaction d'un ouvrage consacré à l'Histoire de la démocratie française, qui essaie de mettre pratiquement en œuvre les préoccupations et les orientations méthodologiques qui sont ici suggérées. Dans Le Moment Guizot (Paris, Gallimard, 1985), j'avais déjà effectué une première tentative, encore limitée et hésitante, dans cette direction de l'histoire conceptuelle du politique, cherchant à prendre mes distances vis-à-vis de l'histoire politique traditionnelle aussi bien que vis-à-vis de ce qu'il est convenu d'appeler l'histoire des idées. À mi-chemin d'un livre dorénavant détaché de moi, et qu'il m'est donc possible de relire d'un ail critique, et d'un ouvrage qui est encore devant moi, cette note de travail reflète le sens d'un effort en train de se faire et ne formule donc en aucune manière les conclusions méthodologiques d'un travail qui aurait cessé de s'interroger sur lui-même.

35. Sur ce débat qui n'a eu que très peu d'échos en France, la bibliographie est immense. Pour s'en faire une idée, on pourra lire deux articles fondamentaux : J. G. A. Pocock, "The History of Political Thought : a Methodological Enquiry ", in Philosophy, Politics and Societies, $2^{\text {nd }}$ series, ed. by Peter LASLETT, Oxford, Oxford University Press, 1962 (Pocock est aussi l'auteur du remarquable The Machiavellian Moment, Princeton, 1975 ; la dimension de cet article ne nous permet pas de discuter la méthode de son ouvrage ni non plus celle du Politics and Vision de Sheldon Woolin, Boston, Little, Brown and Co., 1960 ; ces deux ouvrages ont ouvert une perspective très proche de ce que j'appelle ici l'histoire conceptuelle du politique tout en s'étant donné pour objet une période peut-être trop ample) ; Peter L. JANSSEN : " Political Thought as Traditionnary Action : the Critical Response to Skinner and Pocock ", History and Theory, XXIV, 2,1985 . Tout ce débat mériterait d'être longuement présenté au public français (des milliers de pages lui ont été consacrées en anglais).

36. Ce qui pose, notons-le, le problème du traitement de la modernité comme un champ problématique aux termes relativement constants. Cette question mériterait qu'un débat soit consacré à la pertinence du concept de modernité en philosophie politique. 